\title{
Regression and ANN Models for Electronic Circuit Design
}

\author{
M. I. Dieste-Velasco $\mathbb{D}$, M. Diez-Mediavilla $\mathbb{D}$, and C. Alonso-Tristán $\mathbb{C}$
}

Electromechanical Engineering Department, University of Burgos, Burgos, Spain

Correspondence should be addressed to M. I. Dieste-Velasco; midieste@ubu.es

Received 29 December 2017; Accepted 17 May 2018; Published 16 July 2018

Academic Editor: Laszlo T. Koczy

Copyright $\odot 2018$ M. I. Dieste-Velasco et al. This is an open access article distributed under the Creative Commons Attribution License, which permits unrestricted use, distribution, and reproduction in any medium, provided the original work is properly cited.

\begin{abstract}
This paper presents a methodology to design and to predict the behaviour of electronic circuits, which combines artificial neural networks and design of experiments. This methodology can be used to model output variables in electronic circuits either with similar features to the circuit configuration that is analysed in this study or with more complex configurations in order to improve the process of electronic circuit design.
\end{abstract}

\section{Introduction}

Artificial neural networks (ANNs) are employed in a wide range of applications for optimization, system identification, control and pattern recognition, among others [1]. ANNs have been widely used in several fields of both engineering and technology, and many researchers have analysed their application over the past few years. Among the published research works in the field of ANNs, the research study of Kamar et al. [2] predicted the cooling load, compressor power input, and the performance coefficients of an airconditioning system. Their results showed correlation coefficients that were very close to unity, which indicates that their ANN models predicted the selected performance parameters to a high degree of accuracy [2]. In another study, Xiao et al. [3] used backpropagation neural networks for predicting oxygen dissolved in water with a combination of linear, logsig, and tansig transfer functions. The authors found that the neural networks yielded the most accurate results, in comparison with common prediction methods such as curve fitting and autoregression, among various others [3]. Further examples might include works by Peng et al. [4] in which short-term wind power produced at a wind farm was analysed with ANNs and a hybrid strategy based on both physical and statistical methods [4]. It is also worth mentioning the study in which Karami [5] proposed a method of estimating transient stability analysis in a power system using multilayer perceptrons. The author concluded that the proposed approach was highly suitable for online normalized transient stability margin estimation, because of its accuracy and computational efficiency [5]. Moreover, Srinivasan and Saghir [6] reported the results of modelling thermodiffusion in molten metals, by training neural networks with the LevenbergMarquardt backpropagation algorithm. They concluded that a well-trained neural network can be reliably employed to quantify the thermotransport properties of binary metal alloys [6]. On the other hand, in the research study of Notton et al. [7], the authors determined global solar irradiation on tilted planes, by using multilayer perceptron, feedforward backpropagation, and the Levenberg-Marquardt algorithm [7]. In other studies, Gao et al. [8] proposed a modelling and error compensation approach based on backpropagation neural networks, for an articulated arm coordinate measuring machine. Likewise, Pedro et al. [9] published their design of a neural network for an antilock braking system. They concluded that the NN-based controller provided better results than the generic PID-based controller [9].

In addition to artificial neural networks, fuzzy set theory and their combination have been studied in depth over the past few years [10] and many researchers have investigated their application. For instance, Lovassy et al. [10] proposed fuzzy flip-flop-based neural networks (FNN) as an implementation of multilayer perceptron, in which the network was trained with the Levenberg-Marquardt algorithm. These authors found that this neural network is able to approximate both single and multiple variable functions to a high degree 
of accuracy [10]. Zhang et al. [11] proposed the application of a hierarchical fuzzy neural network as a new type of fault diagnosis for an asynchronous motor, which performed effective diagnosis of both single and multiple faults [11]. Yang et al. [12] employed FNNs to provide an efficient means of detecting and evaluating concrete strength, and Yang et al. [13] combined fuzzy theory and artificial neural network techniques to evaluate and to analyse the safety status of an oil depot [13]. In further research studies, Lovassy et al. [14] reported an analysis of the behaviour of an FPGA implementation of the FNNs and went on to state that their FPGA implementation provided a safe and hazardless solution in environments where observation might be distorted by a component of noise [14].

In other research studies that concern the applicability of ANNs, Djeffal et al. [15] showed the applicability of ANNs in the simulation of nanoscale CMOS circuits, training the ANN with a backpropagation algorithm. Moreover, Mohagheghi et al. [16] employed neural networks for the monitoring of electronic power circuits. Among their conclusions, they mentioned that, with a proper and a systematic selection of design parameters and sufficient training data, a neural network can learn to model any nonlinear system to a high level of accuracy [16].

This present research study compares the results provided by ANNs with those of a conventional regression. Moreover, it examines how the ANN is employed to obtain the main effect plots and the interaction effect plots, in order to determine the influence of the input parameters on the circuit response. It is shown that ANNs can be used to analyse electronic circuits and to optimize their performance. This methodology may also be used to model other output variables in electronic circuits with more complex configurations, in order to improve the process of electronic circuits design.

\section{Modelling of Circuit Parameters}

A bipolar transistor, configured as a common emitter amplifier, is selected as an example application to show the proposed methodology. The gain voltage and the relation between the collector current and base current $\left(I_{C} / I_{B}\right)$ of the transistor are determined, following a full factorial design of experiments when the electrical resistances in the circuit are varied, from software simulations. These output values are then used to train an artificial neural network, in order to model both the gain voltage and the $I_{\mathrm{C}} / I_{\mathrm{B}}$ as a function of the electrical resistances in the amplifier circuit. Once the artificial neural network is trained, it can then be used to obtain both the gain voltage and the $I_{\mathrm{C}} / I_{\mathrm{B}}$, when the electrical resistances are varied, with no further need for additional simulations. A $3^{4}$ factorial design of experiments (DOE) was selected, which corresponds to 81 possible combinations of the input parameters (electrical resistances).

Table 1 shows the range of variation of the electrical resistances that form part of the electrical circuit shown in Figure 1. This circuit is analysed in this present study by training a neural network and then using the results to obtain the main effect plots and the interaction effect plots. That is,
TABLE 1: Electrical resistance values.

\begin{tabular}{lccc}
\hline & Low & Nominal & High \\
\hline$R_{1}(\mathrm{k} \Omega)$ & 9.00 & 12.0 & 15.0 \\
$R_{2}(\mathrm{k} \Omega)$ & 2.25 & 3.0 & 3.8 \\
$R_{3}(\mathrm{k} \Omega)$ & 3.00 & 4.0 & 5.0 \\
$R_{4}(\mathrm{k} \Omega)$ & 1.50 & 2.0 & 2.5 \\
\hline
\end{tabular}

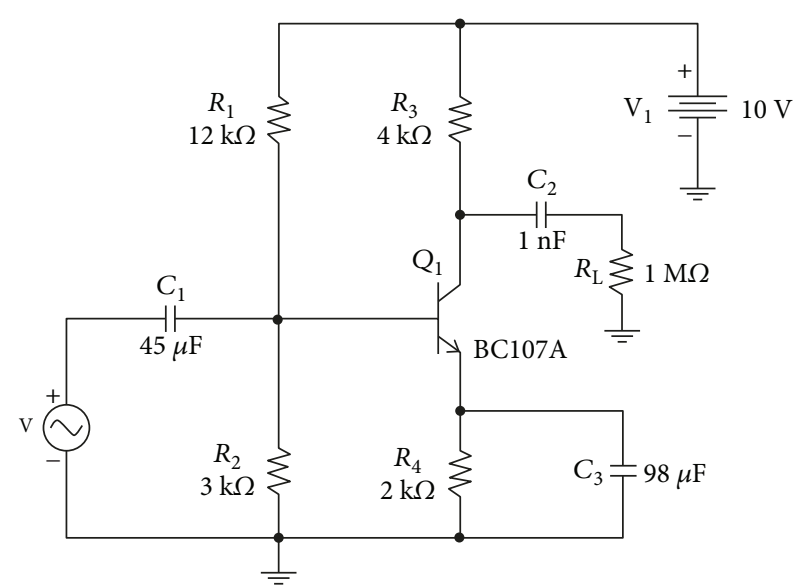

FIgURE 1: Nominal values of the amplifier circuit.

Table 1 shows the electrical resistance levels, which were selected following a full factorial DOE, based on raising and lowering the nominal value of the electrical resistances by $25 \%$ (from the centre value). The number of resistance values which could be employed, following this methodology, may be higher than three with the aim of obtaining a large number of data to train the ANN. Nevertheless, in this study, we have considered that three levels of variation is a high enough number to obtain significant results.

Figure 1 shows the nominal values of the amplifier circuit employed in this study, where $R_{j}$ are the electrical resistances to be varied and $V$ is a $20 \mathrm{mV}$ and $1 \mathrm{kHz}$ sinusoidal voltage source.

The values shown in Figure 1 may be modified with the data shown in Table 1, following a full-factorial DOE. After simulation of these electronic circuits, it will be possible to determine the gain voltage and the relation between both the collector and base currents of the transistor.

\section{ANN and Regression Modelling}

As was previously mentioned, simulations made it possible to determine the output data that correspond to all of the possible combinations of electrical resistances shown in Table 1 . This will lead to different transistor polarizations that will induce variations in the gain voltage and in the $I_{\mathrm{C}} / I_{\mathrm{B}}$. By using the data shown in Table 2, a neural network can then be trained to predict both the $I_{\mathrm{C}} / I_{\mathrm{B}}$ and the gain voltage of the amplifier circuit.

The ANN employed in this present study is composed of a hidden layer and an output layer. Figure 2 shows the configuration of the neural network used for modelling the 
TABLE 2: Gain voltage $\Delta(V)$ and $I_{\mathrm{C}} / I_{\mathrm{B}}$ results.

\begin{tabular}{|c|c|c|c|c|c|c|}
\hline$n$ & $R_{1}$ & $R_{2}$ & $R_{3}$ & $R_{4}$ & $\frac{I_{\mathrm{C}}}{I_{\mathrm{B}}}$ & $\Delta(V)$ \\
\hline 1 & 15.00 & 3.00 & 4.00 & 2.50 & 149.41 & 2.33 \\
\hline 2 & 15.00 & 2.25 & 5.00 & 1.50 & 150.66 & 3.12 \\
\hline 3 & 9.00 & 2.25 & 4.00 & 1.50 & 162.94 & 5.04 \\
\hline 4 & 12.00 & 3.80 & 4.00 & 1.50 & 166.54 & 6.24 \\
\hline 5 & 15.00 & 3.00 & 3.00 & 2.50 & 149.84 & 1.76 \\
\hline 6 & 12.00 & 2.25 & 5.00 & 1.50 & 156.84 & 4.52 \\
\hline 7 & 9.00 & 2.25 & 5.00 & 1.50 & 162.25 & 6.29 \\
\hline 8 & 9.00 & 3.80 & 3.00 & 2.50 & 163.44 & 3.93 \\
\hline 9 & 9.00 & 3.80 & 5.00 & 2.00 & 164.41 & 5.36 \\
\hline 10 & 15.00 & 3.80 & 5.00 & 2.00 & 157.53 & 4.83 \\
\hline 11 & 15.00 & 3.80 & 3.00 & 2.50 & 154.99 & 2.33 \\
\hline 12 & 9.00 & 3.80 & 3.00 & 1.50 & 171.68 & 5.78 \\
\hline 13 & 15.00 & 3.80 & 4.00 & 2.00 & 158.30 & 3.82 \\
\hline 14 & 9.00 & 3.80 & 3.00 & 2.00 & 167.19 & 4.84 \\
\hline 15 & 9.00 & 3.00 & 3.00 & 2.00 & 163.91 & 3.87 \\
\hline 16 & 15.00 & 3.80 & 4.00 & 1.50 & 163.10 & 5.05 \\
\hline 17 & 12.00 & 3.80 & 4.00 & 2.50 & 158.32 & 3.98 \\
\hline 18 & 15.00 & 3.80 & 5.00 & 1.50 & 162.04 & 6.18 \\
\hline 19 & 15.00 & 3.00 & 3.00 & 2.00 & 153.98 & 2.17 \\
\hline 20 & 9.00 & 2.25 & 3.00 & 1.50 & 163.98 & 3.82 \\
\hline 21 & 9.00 & 3.00 & 4.00 & 2.50 & 159.14 & 4.19 \\
\hline 22 & 9.00 & 2.25 & 4.00 & 2.00 & 158.11 & 3.84 \\
\hline 23 & 9.00 & 2.25 & 5.00 & 2.00 & 157.36 & 4.77 \\
\hline 24 & 12.00 & 3.00 & 3.00 & 2.00 & 158.84 & 2.85 \\
\hline 25 & 12.00 & 2.25 & 5.00 & 2.50 & 147.51 & 2.68 \\
\hline 26 & 12.00 & 2.25 & 5.00 & 2.00 & 151.44 & 3.34 \\
\hline 27 & 15.00 & 2.25 & 5.00 & 2.00 & 145.60 & 2.38 \\
\hline 28 & 12.00 & 2.25 & 4.00 & 1.50 & 157.04 & 3.48 \\
\hline 29 & 9.00 & 3.00 & 4.00 & 2.00 & 162.84 & 5.11 \\
\hline 30 & 9.00 & 3.80 & 5.00 & 2.50 & 161.27 & 5.70 \\
\hline 31 & 9.00 & 3.00 & 5.00 & 1.50 & 165.76 & 5.68 \\
\hline 32 & 9.00 & 3.80 & 4.00 & 2.00 & 165.80 & 5.79 \\
\hline 33 & 12.00 & 2.25 & 3.00 & 1.50 & 157.72 & 2.63 \\
\hline 34 & 15.00 & 2.25 & 4.00 & 1.50 & 151.12 & 2.50 \\
\hline 35 & 9.00 & 2.25 & 3.00 & 2.50 & 154.78 & 2.32 \\
\hline 36 & 9.00 & 2.25 & 5.00 & 2.50 & 153.60 & 3.86 \\
\hline 37 & 12.00 & 2.25 & 3.00 & 2.00 & 152.44 & 2.00 \\
\hline 38 & 12.00 & 2.25 & 4.00 & 2.00 & 151.94 & 2.65 \\
\hline 39 & 15.00 & 3.00 & 5.00 & 2.00 & 152.88 & 3.60 \\
\hline 40 & 12.00 & 3.00 & 3.00 & 2.50 & 154.75 & 2.30 \\
\hline 41 & 15.00 & 3.00 & 5.00 & 2.50 & 148.98 & 2.92 \\
\hline 42 & 12.00 & 3.00 & 3.00 & 1.50 & 163.94 & 3.74 \\
\hline 43 & 12.00 & 3.00 & 5.00 & 2.50 & 153.58 & 3.85 \\
\hline 44 & 12.00 & 3.80 & 3.00 & 1.50 & 167.94 & 4.85 \\
\hline 45 & 15.00 & 2.25 & 3.00 & 2.00 & 146.28 & 1.44 \\
\hline 46 & 12.00 & 3.00 & 4.00 & 2.50 & 154.17 & 3.09 \\
\hline 47 & 12.00 & 2.25 & 3.00 & 2.50 & 148.28 & 1.61 \\
\hline 48 & 12.00 & 3.80 & 5.00 & 2.00 & 161.05 & 6.02 \\
\hline 49 & 9.00 & 2.25 & 3.00 & 2.00 & 158.87 & 2.85 \\
\hline
\end{tabular}

TABle 2: Continued.

\begin{tabular}{ccccccc}
\hline$n$ & $R_{1}$ & $R_{2}$ & $R_{3}$ & $R_{4}$ & $\frac{I_{\mathrm{C}}}{I_{\mathrm{B}}}$ & $\Delta(V)$ \\
\hline 50 & 12.00 & 3.80 & 5.00 & 1.50 & 165.15 & 5.96 \\
51 & 9.00 & 3.00 & 3.00 & 2.50 & 159.98 & 3.16 \\
52 & 15.00 & 2.25 & 3.00 & 1.50 & 151.59 & 1.89 \\
53 & 12.00 & 2.25 & 4.00 & 2.50 & 147.89 & 2.14 \\
54 & 9.00 & 3.80 & 5.00 & 1.50 & 145.53 & 4.83 \\
55 & 15.00 & 2.25 & 4.00 & 2.00 & 145.94 & 1.91 \\
56 & 15.00 & 2.25 & 4.00 & 2.50 & 141.87 & 1.55 \\
57 & 12.00 & 3.80 & 3.00 & 2.00 & 163.07 & 3.65 \\
58 & 12.00 & 3.00 & 5.00 & 2.00 & 157.33 & 4.76 \\
59 & 12.00 & 3.00 & 4.00 & 1.50 & 162.90 & 5.03 \\
60 & 12.00 & 3.80 & 5.00 & 2.50 & 157.53 & 4.93 \\
61 & 15.00 & 2.25 & 5.00 & 2.50 & 141.61 & 1.93 \\
62 & 9.00 & 3.00 & 3.00 & 1.50 & 168.73 & 5.14 \\
63 & 9.00 & 3.00 & 5.00 & 2.00 & 161.76 & 6.10 \\
64 & 15.00 & 3.00 & 4.00 & 1.50 & 158.47 & 3.78 \\
65 & 9.00 & 3.00 & 5.00 & 2.50 & 158.30 & 5.09 \\
66 & 12.00 & 3.80 & 4.00 & 2.00 & 162.06 & 4.91 \\
67 & 15.00 & 3.80 & 3.00 & 1.50 & 164.15 & 3.78 \\
68 & 15.00 & 3.00 & 3.00 & 1.50 & 159.23 & 2.85 \\
69 & 9.00 & 3.80 & 4.00 & 1.50 & 169.76 & 5.33 \\
70 & 9.00 & 2.25 & 4.00 & 2.50 & 154.19 & 3.07 \\
71 & 15.00 & 3.80 & 5.00 & 2.50 & 153.80 & 3.91 \\
72 & 15.00 & 3.00 & 5.00 & 1.50 & 157.72 & 4.76 \\
73 & 12.00 & 3.00 & 5.00 & 1.50 & 158.61 & 6.12 \\
74 & 15.00 & 3.80 & 3.00 & 2.00 & 159.06 & 2.88 \\
75 & 9.00 & 3.80 & 4.00 & 2.50 & 162.35 & 5.16 \\
76 & 15.00 & 3.00 & 4.00 & 2.00 & 153.43 & 2.88 \\
77 & 15.00 & 2.25 & 3.00 & 2.50 & 142.14 & 1.16 \\
78 & 9.00 & 3.00 & 4.00 & 1.50 & 167.24 & 6.15 \\
79 & 12.00 & 3.00 & 4.00 & 2.00 & 158.08 & 3.83 \\
80 & 15.00 & 3.80 & 4.00 & 2.50 & 154.39 & 3.09 \\
81 & 12.00 & 3.80 & 3.00 & 2.50 & 159.11 & 2.96 \\
\hline & & & & & &
\end{tabular}

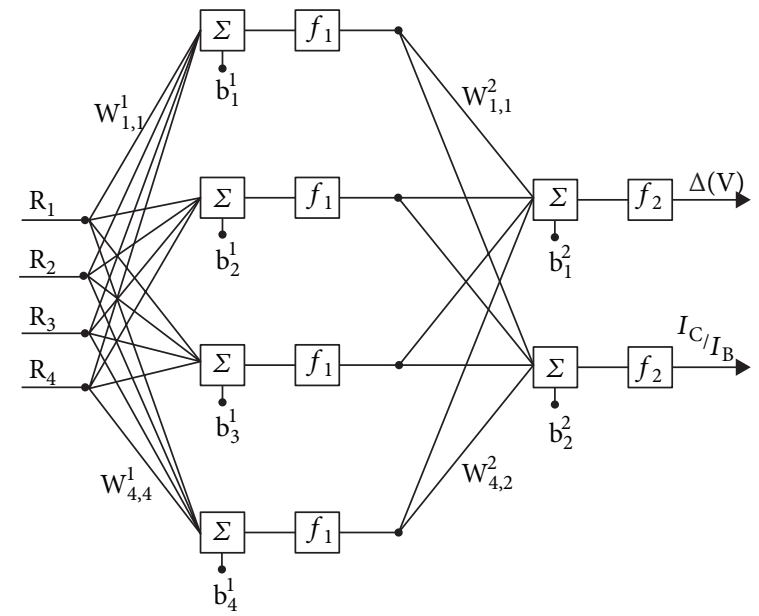

Figure 2: ANN structure. 

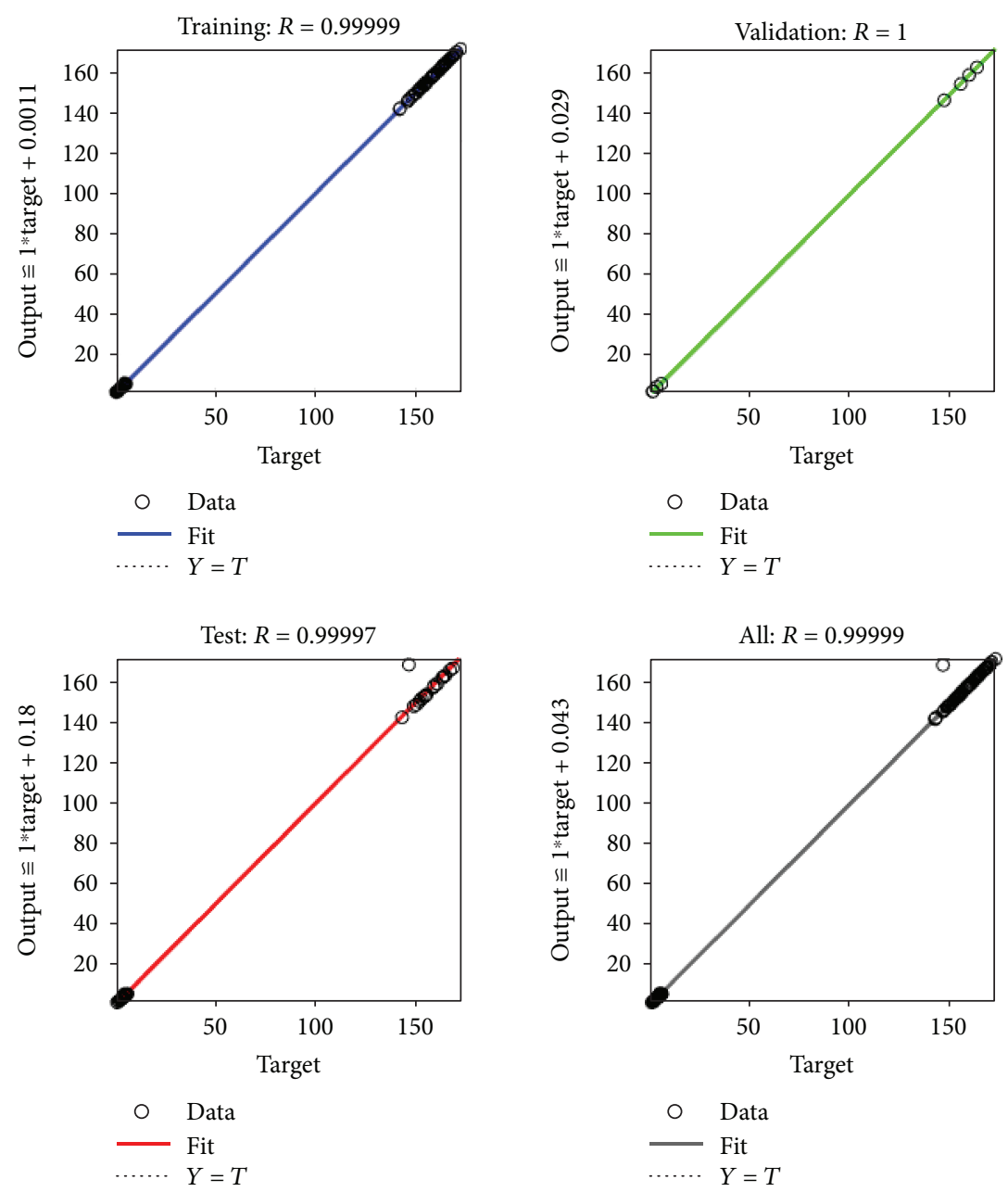

FIGURE 3: Results using the ANN.

behaviour of both the $I_{\mathrm{C}} / I_{\mathrm{B}}$ and the $\Delta(V)$. The results dealing with the ANN were calculated with the Neural Network Toolbox $^{\mathrm{TM}}$ of MATLAB ${ }^{\circledR}$ (MathWorks Inc.).

As may be seen, the same ANN was employed to model both outputs. Following a trial-and-error process, a hidden layer with four neurons was selected for modelling the values of both $I_{\mathrm{C}} / I_{\mathrm{B}}$ and $\Delta(V)$.

Figure 2 shows the neural network configuration, which corresponds to four inputs $\left(R_{1}, R_{2}, R_{3}\right.$, and $\left.R_{4}\right)$, four neurons in the hidden layer, and two outputs $\left(I_{\mathrm{C}} / I_{\mathrm{B}}\right.$ and $\left.\Delta(V)\right)$. A DOE was used to train the ANN network, in order to obtain the main effect plot and the interaction effect plot. MSE was used as a performance index.

A log-sigmoid, defined by (1), was used as the transfer function $\left(f_{1}\right)$ for the hidden layer, and a pure linear function, shown in (2), was used for the output layer $\left(f_{2}\right)$, while a Levenberg-Marquardt algorithm was used to train the neural network.

$$
\begin{aligned}
& f_{1}(x)=\frac{1}{1+e^{-x}}, \\
& f_{2}(x)=x .
\end{aligned}
$$

Equations (3) and (4) may be obtained from Figure 2. These equations allow us to determine both the $I_{\mathrm{C}} / I_{\mathrm{B}}$ and the $\Delta(V)$ as a function of the input parameters. As function $f_{2}$ is purely linear, (4) can be written as follows:

$$
\begin{aligned}
& \text { output }_{1}=f_{1}\left(W_{1} * \text { inputs }+b_{1}\right), \\
& \text { output }_{2}=W_{2} * f_{1}\left(W_{1} * \text { inputs }+b_{1}\right)+b_{2} .
\end{aligned}
$$

As previously mentioned, simulation results shown in Table 2 were used to train, to validate, and to test the neural network $(70 \%, 15 \%$, and $15 \%)$, respectively. Figure 3 shows the values obtained to measure the correlation between outputs and targets using the ANN. The performance index is also shown in Figure 4.

Figure 4 shows the training performance as well as the error histogram and the regression plot for the ANN considered in this present study. A conventional regression analysis was also performed using the whole set of experiments. The adjusted $R$-squared statistic indicated that the regression model explained $88 \%$ of the variability in $I_{\mathrm{C}} / I_{\mathrm{B}}$ and $90 \%$ of the variability in $\Delta(V)$. As can be observed, these values are much smaller than those obtained with the ANN. 

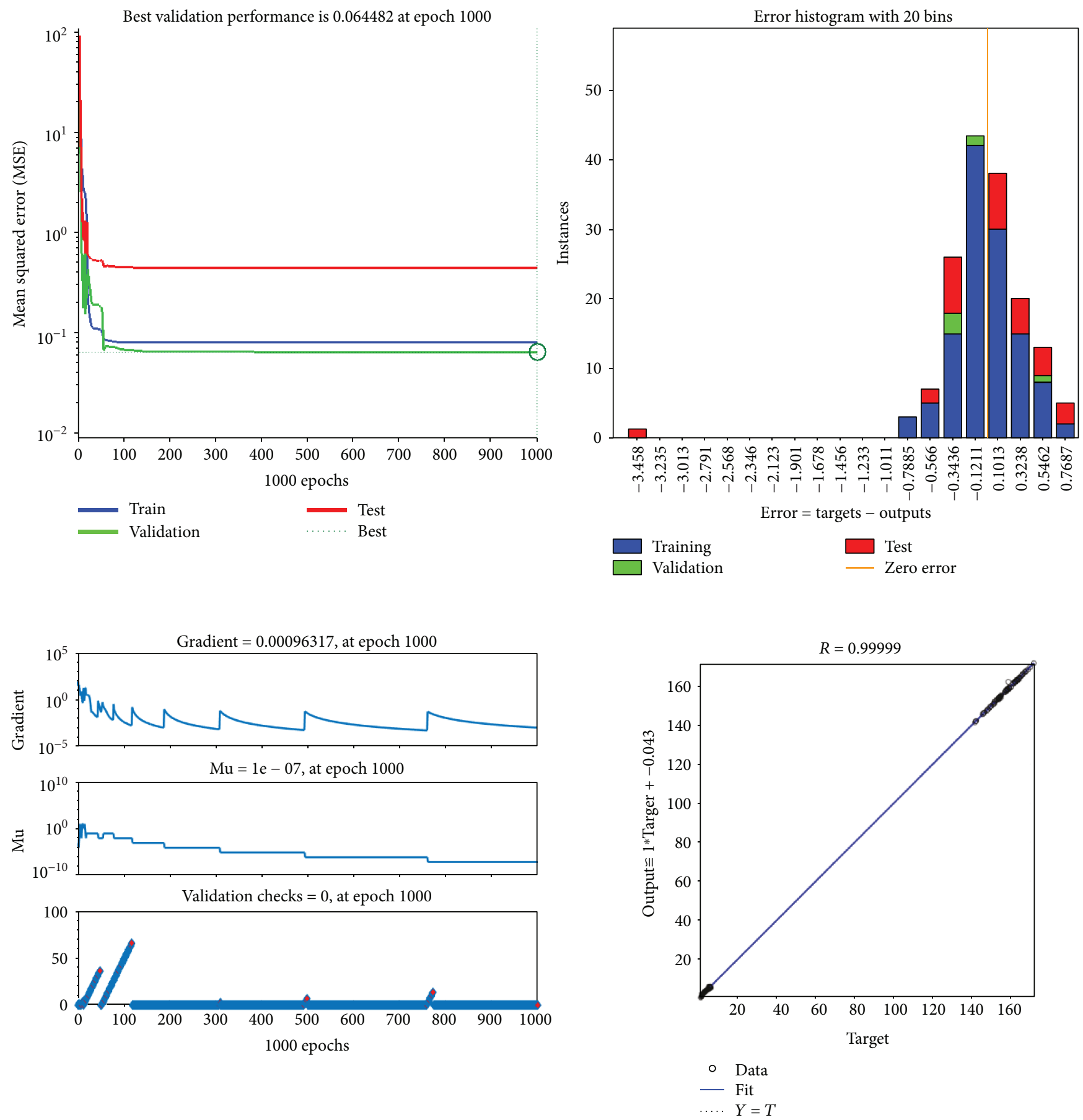

FIGURE 4: Neural network training state and error diagrams.

TABLE 3: First layer weights and biases.

\begin{tabular}{lcccc}
\hline \multicolumn{4}{c}{ Weights } & Biases \\
\hline-0.3842 & 0.2583 & -0.4092 & -0.4423 & -0.2399 \\
-0.3798 & 0.2564 & -0.3915 & -0.4369 & -0.2456 \\
-0.8592 & 1.6675 & 0.0988 & -0.3028 & 2.3048 \\
3.7471 & -3.5843 & -3.7366 & 3.1795 & 18.8845 \\
\hline
\end{tabular}

TABLE 4: Second layer weights and biases.

\begin{tabular}{|c|c|c|c|c|}
\hline \multicolumn{4}{|c|}{ Weights } & \multirow{2}{*}{$\begin{array}{c}\text { Biases } \\
-166.7892\end{array}$} \\
\hline-57.2214 & 60.3744 & 0.9869 & 164.7291 & \\
\hline-174.2275 & 178.5871 & 0.9639 & 81.1056 & -83.5416 \\
\hline
\end{tabular}



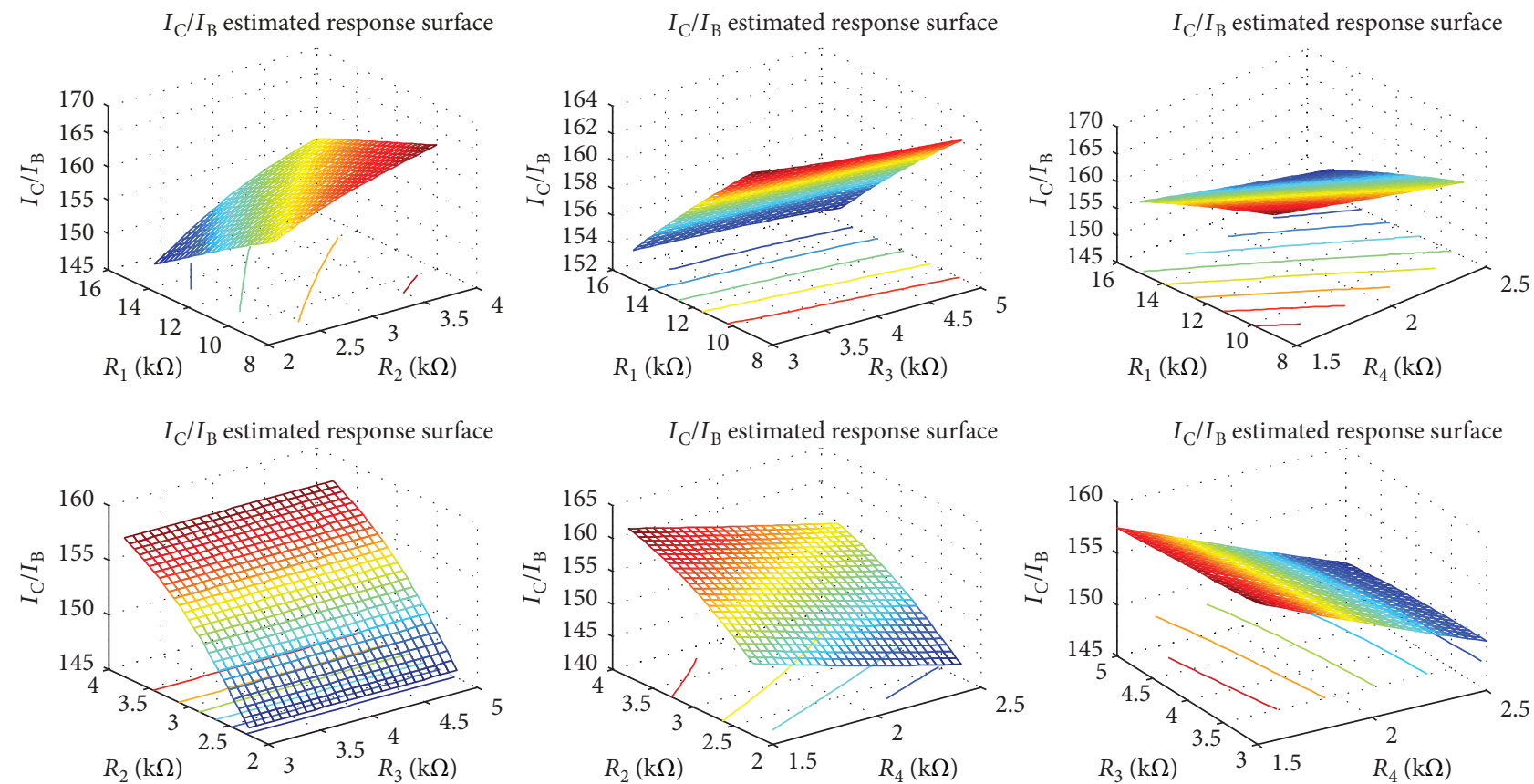

Figure 5: Estimated ANN response surface for the $I_{\mathrm{C}} / I_{\mathrm{B}}$.

Equation (5) show the regression polynomials for the $I_{\mathrm{C}} / I_{\mathrm{B}}$ and the $\Delta(V)$.

$$
\begin{aligned}
\frac{I_{\mathrm{C}}}{I_{\mathrm{B}}}= & 187.197-3.145 * R_{1}+12.026 * R_{2}-1.281 \\
& * R_{3}-14.881 * R_{4}+0.781 * R_{1} * R_{2}+0.295 \\
& * R_{1} * R_{3}-0.560 * R_{1} * R_{4}-1.192 * R_{2} * R_{3} \\
& +2.275 * R_{2} * R_{4}+1.848 * R_{3} * R_{4}-0.031 \\
& * R_{1}^{2}-2.530 * R_{2}^{2}-0.457 * R_{3}^{2}-0.085 * R_{4}^{2} \\
\Delta(V)= & 3.093-0.528 * R_{1}+2.055 * R_{2}+1.480 * R_{3} \\
& -2.840 * R_{4}+0.086 * R_{1} * R_{2}+0.013 * R_{1} \\
& * R_{3}-0.025 * R_{1} * R_{4}-0.018 * R_{2} * R_{3} \\
& +0.072 * R_{2} * R_{4}+0.020 * R_{3} * R_{4}-0.001 \\
& * R_{1}^{2}-0.335 * R_{2}^{2}-0.108 * R_{3}^{2}+0.344 * R_{4}^{2} .
\end{aligned}
$$

Tables 3 and 4 show the weights and biases of the ANN. Once determined, the ANN may be used to predict the output values. Moreover, it is possible to employ the ANN to obtain response surfaces for the $I_{\mathrm{C}} / I_{\mathrm{B}}$ and the $\Delta(V)$ as well as to analyse the effect of the input variables on these parameters. As previously shown, the results of the neural network are of higher accuracy than the results of the regression analysis. The ANN therefore has many advantages over traditional methodologies that analyse the DOE from mean values. However, the models obtained with the ANN are often more complex than those obtained by regression analysis.

\section{Discussion}

Figures 5 and 6 show the neural network response surfaces for both the $I_{\mathrm{C}} / I_{\mathrm{B}}$ and the $\Delta(V)$. Once the weight and bias parameters are obtained, it is possible to plot the response surfaces. For example, the response surface $I_{\mathrm{C}} / I_{\mathrm{B}}=f\left(R_{1}, R_{2}\right)$ is obtained when both $R_{1}$ and $R_{2}$ are varied, while $R_{3}$ and $R_{4}$ are kept at their central values.

The same procedure can be used to determine the main effect plot and the interaction effect plot. In this case, to obtain the main effect plot, one input parameter is varied while the others are kept at their central values, as shown by Figure 7. The interaction plot between two parameters is obtained by considering the higher and the lower values of one parameter, while the other is modified in its variation range and the rest of the parameters are kept at their central values, as shown in Figures 8 and 9. In this way, it is possible to obtain the interaction effect plot and the main effect plot using the ANN, where the inputs have to be selected according to the aforementioned procedure.

As mentioned earlier, the influence of the parameters on both the $I_{\mathrm{C}} / I_{\mathrm{B}}$ and the $\Delta(V)$ may be analysed by means of the interaction effect plot and the main effect plot. These plots can be obtained by determining the outputs provided by the neural network when appropriate inputs are considered.

Figure 7 shows the main effect plot which has been normalized from the expressions shown in (6), in which the response variables were obtained from the data given by the previously determined artificial neuronal network.

$$
\frac{I_{\mathrm{c}}}{I_{\mathrm{b}_{\text {normalized }}}}=\frac{I_{R_{j}} / I_{b_{R j}}-\min \left(I_{c} / I_{b_{R_{1}}, \ldots, R_{4}}\right)}{\max \left(I_{c} / I_{b_{R_{1}, \ldots, R_{4}}}\right)-\min \left(I_{c} / I_{b_{R_{1}, \ldots, R_{4}}}\right)},
$$

$$
\Delta(V)_{\text {normalized }_{R_{j}}}=\frac{\Delta(V)_{R_{j}}-\min \left(\Delta(V)_{R_{1}, \ldots, R_{4}}\right)}{\max \left(\Delta(V)_{R_{1}, \ldots, R_{4}}\right)-\min \left(\Delta(V)_{R_{1}, \ldots, R_{4}}\right)},
$$



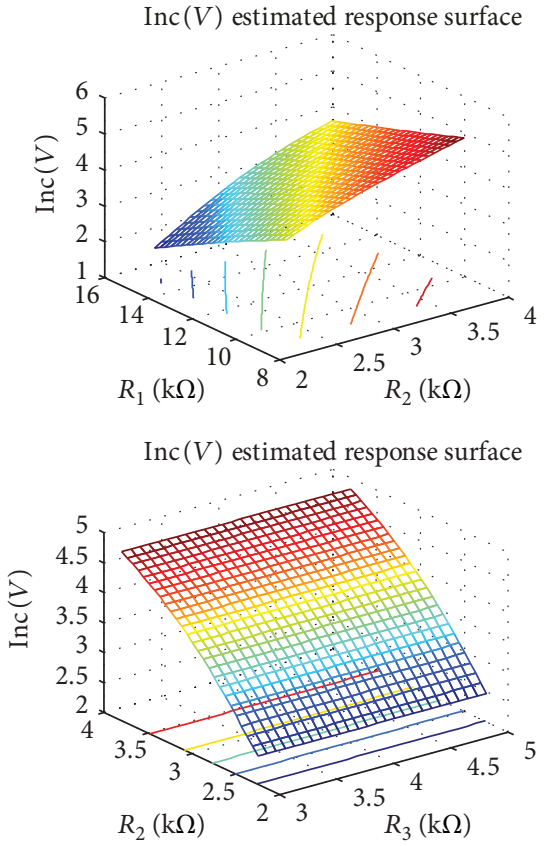

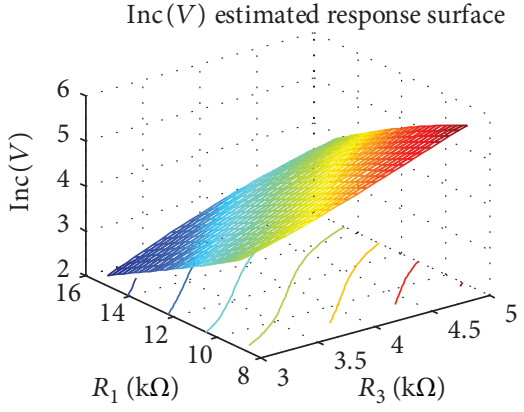

$\operatorname{Inc}(V)$ estimated response surface

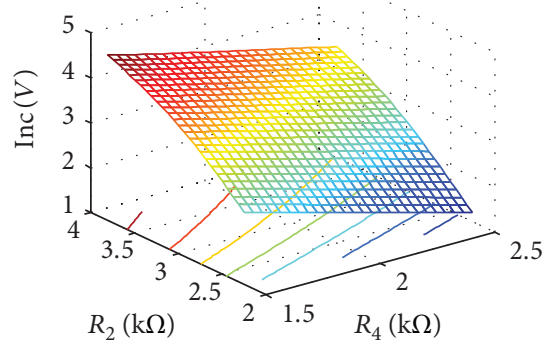

$\operatorname{Inc}(V)$ estimated response surface

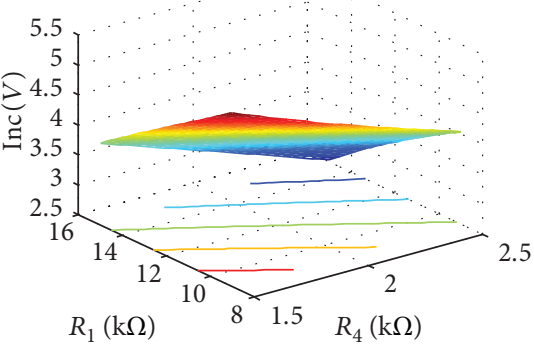

$\operatorname{Inc}(V)$ estimated response surface

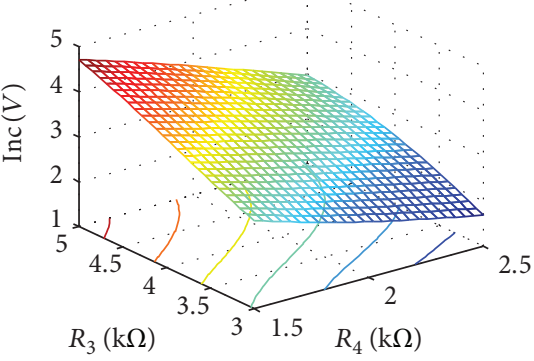

Figure 6: Estimated ANN response surface for the $\Delta(V)$.

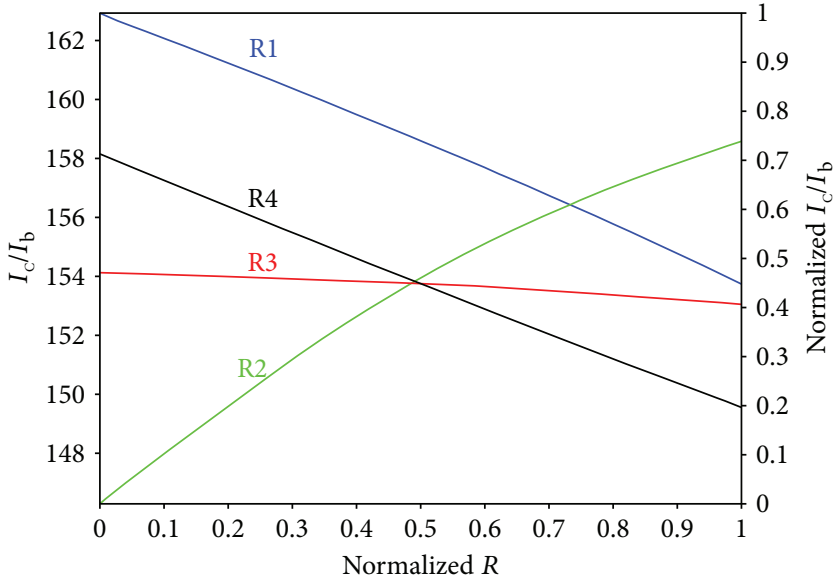

(a)

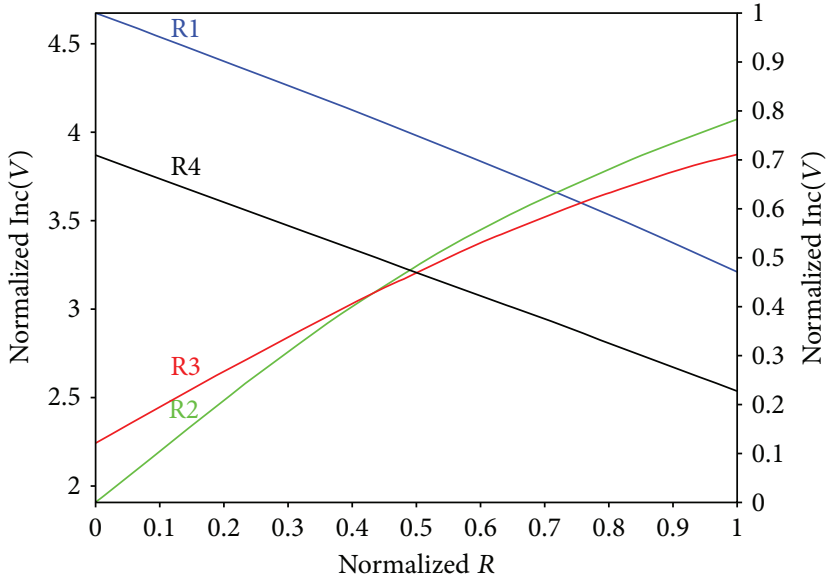

(b)

FIgUre 7: Main effect plots showing the $I_{\mathrm{C}} / I_{\mathrm{B}}$ and the $\Delta(V)$ values obtained with the ANN.

$$
\operatorname{normalized}_{R_{j}}=\frac{R_{j}-\min \left(R_{j}\right)}{\max \left(R_{j}\right)-\min \left(R_{j}\right)} .
$$

As can be observed in Figure 7(a), for this present study, when $R_{2}-R_{4}$ are kept at their central values, the $I_{\mathrm{C}} / I_{\mathrm{B}}$ reaches a maximum value, if $R_{1}$ is kept at its minimum value. The same behaviour is observed for $R_{3}$ and $R_{4}$. On the contrary, $I_{\mathrm{C}} / I_{\mathrm{B}}$ reaches a maximum value, if $R_{2}$ is kept at its maximum value, while $R_{1}, R_{3}$, and $R_{4}$ are kept at their central values.

As may also be observed in Figure 7(a), $R_{2}$ is the parameter which affects the $I_{\mathrm{C}} / I_{\mathrm{B}}$ more than any other, followed by $R_{1}$ and $R_{4}$. The collector resistance $\left(R_{3}\right)$ parameter affects the
$I_{\mathrm{C}} / I_{\mathrm{B}}$ less than any other, and its influence is much smaller than the influence of the other three electrical resistances.

In the case of the gain voltage, as can also be observed in Figure 7(b), the value of $\Delta(V)$ reaches a maximum, when $R_{2}-R_{4}$ are kept at their central values, if $R_{1}$ is kept at its minimum value. The same behaviour may be observed for $R_{4}$. On the contrary, the value of $\Delta(V)$ reaches a maximum, if either $R_{2}$ or $R_{3}$ is kept at its maximum value, while the rest of the electrical resistances are kept at their central values. As may also be observed in Figure 7(b), the most influential parameters are $R_{2}$ and $R_{3}$, followed by $R_{1}$ and $R_{4}$.

As can be observed in Figure 8, $R_{2}$ exhibits a different behaviour, with respect to $R_{1}$ and to the behaviour shown by $R_{3}$ and $R_{4}$. The same trend is observed in the three electrical resistors $R_{2}-R_{4}$, when they are kept at either minimum or 

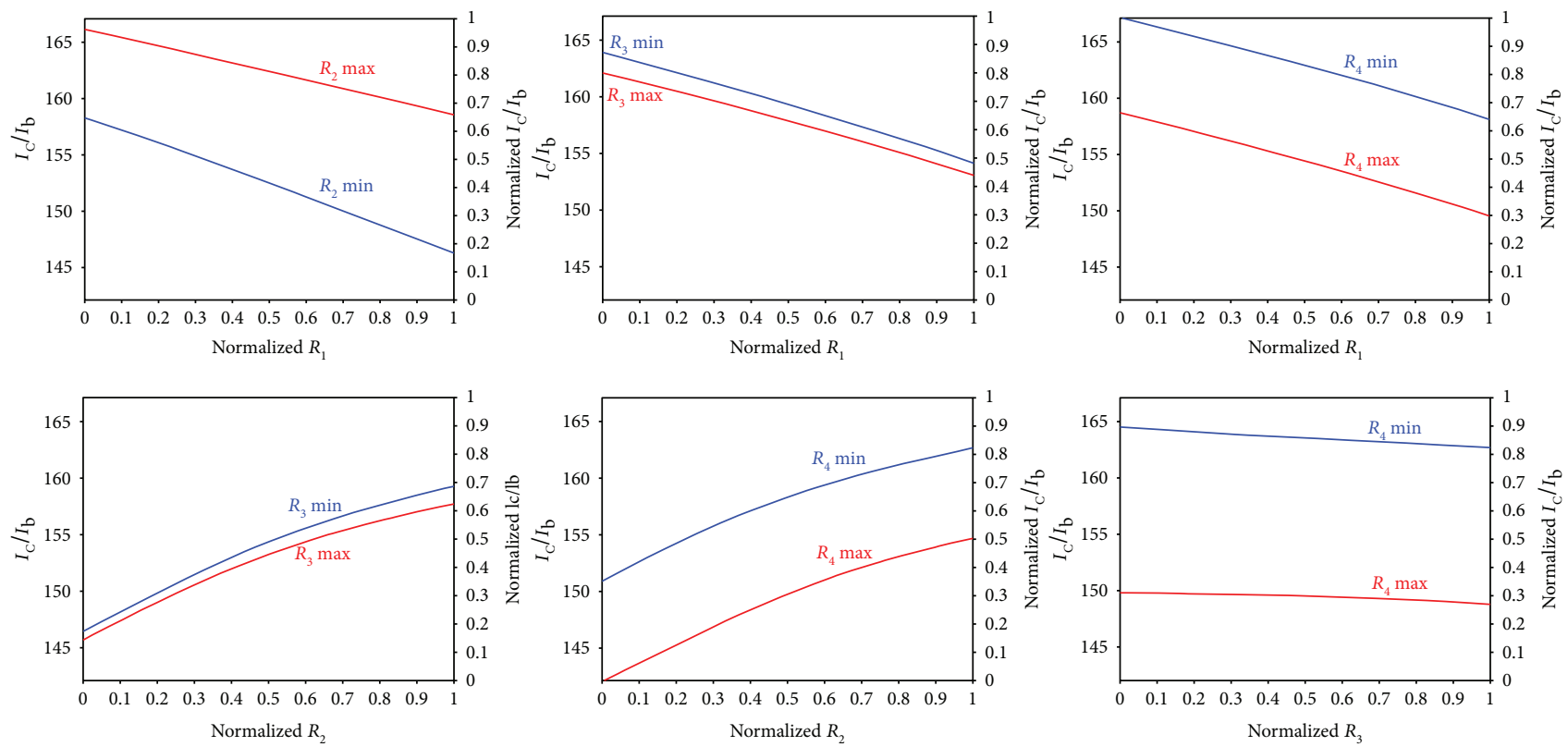

Figure 8: Interaction effect plot showing the $I_{\mathrm{C}} / I_{\mathrm{B}}$ values obtained with the ANN.
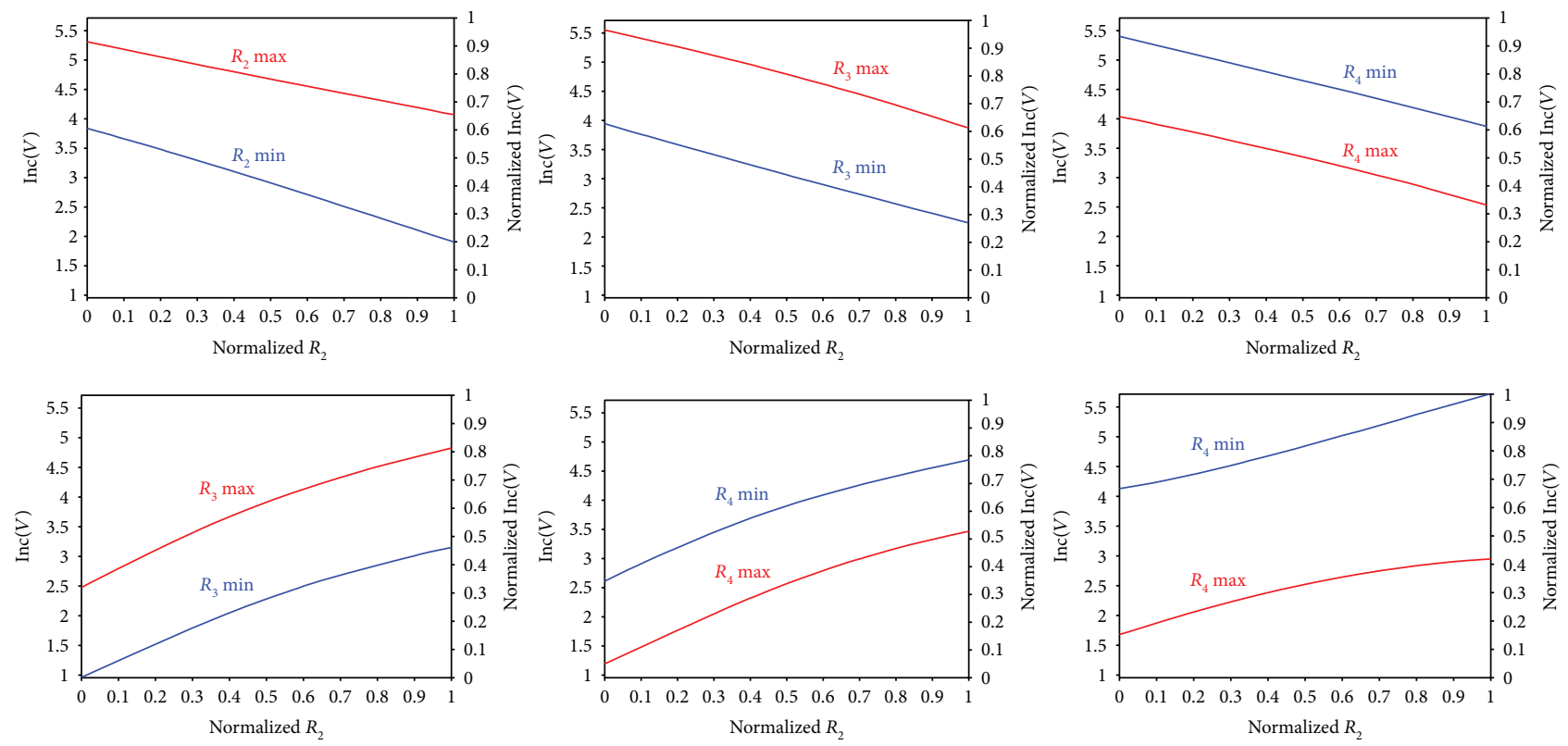

FIGURE 9: Interaction effect plots for the $\Delta(V)$ obtained with the ANN.

maximum values and $R_{1}$ is varied, that is, the lower the electrical resistances, the higher the $I_{C} / I_{\mathrm{B}}$. The influence of $R_{3}$ and $R_{4}$ on $R_{2}$ is approximately the same, while the interaction between $R_{3}$ and $R_{4}$ is much lower.

As can be observed in Figure 9, the behaviour of $R_{4}$ in relation to $R_{1}$ differs in relation to $R_{2}$ and $R_{3}$. The same trend is observed in the three electrical resistors $R_{2}-R_{4}$, when they are kept at either minimum or maximum values, while $R_{1}$ is varied: the lower the electrical resistance, the higher the $\Delta$ $(V)$. The influence of $R_{3}$ and $R_{4}$ on $R_{2}$ is very similar, but its behaviour is different, the interaction between the collector resistance $\left(R_{3}\right)$ and the emitter resistance $\left(R_{4}\right)$ being much lower than the other interactions between the electrical resistances of the amplifier circuit.

\section{Conclusions}

In this present study, a neural network comprising four inputs, a hidden layer of four neurons, and two outputs has been used to model output variables in an electronic circuit. The findings have shown that a factorial DOE combined with an ANN can model the behaviour of $I_{C} / I_{B}$ and $\Delta(V)$ in an efficient way. Higher accuracy was obtained with the ANN than with conventional regression techniques. 
The methodology proposed in this study therefore has the potential to assist the analytical design of electronic circuits and their actual implementation, either with similar features to that shown in this present study or with more complex configurations.

\section{Conflicts of Interest}

There are no conflicts of interest regarding the publication of this paper.

\section{References}

[1] Y. Jiang, C. Yang, J. Na, G. Li, Y. Li, and J. Zhong, "A brief review of neural networks based learning and control and their applications for robots," Complexity, vol. 2017, Article ID 1895897, 14 pages, 2017.

[2] H. M. Kamar, R. Ahmad, N. B. Kamsah, and A. F. Mohamad Mustafa, "Artificial neural networks for automotive airconditioning systems performance prediction," Applied Thermal Engineering, vol. 50, no. 1, pp. 63-70, 2013.

[3] Z. Xiao, L. Peng, Y. Chen, H. Liu, J. Wang, and Y. Nie, “The dissolved oxygen prediction method based on neural network," Complexity, vol. 2017, Article ID 4967870, 6 pages, 2017.

[4] H. Peng, F. Liu, and X. Yang, "A hybrid strategy of short term wind power prediction," Renewable Energy, vol. 50, pp. 590595, 2013.

[5] A. Karami, "Power system transient stability margin estimation using neural networks," International Journal of Electrical Power \& Energy Systems, vol. 33, no. 4, pp. 983-991, 2011.

[6] S. Srinivasan and M. Z. Saghir, "Modeling of thermotransport phenomenon in metal alloys using artificial neural networks," Applied Mathematical Modelling, vol. 37, no. 5, pp. 2850-2869, 2013.

[7] G. Notton, C. Paoli, L. Ivanova, S. Vasileva, and M. L. Nivet, "Neural network approach to estimate 10-min solar global irradiation values on tilted planes," Renewable Energy, vol. 50, pp. 576-584, 2013.

[8] G. Gao, H. Zhang, H. San, X. Wu, and W. Wang, "Modeling and error compensation of robotic articulated arm coordinate measuring machines using BP neural network," Complexity, vol. 2017, Article ID 5156264, 8 pages, 2017.

[9] J. O. Pedro, O. T. C. Nyandoro, and S. John, "Neural network based feedback linearisation slip control of an anti-lock braking system," in Proceedings of the 7th Asian Control Conference, pp. 1251-1257, Hong Kong, China, August 2009.

[10] R. Lovassy, L. T. Kóczy, and L. Gál, "Function approximation capability of a novel fuzzy flip-flop based neural network," in Proceedings of the 2009 International Joint Conference on Neural Networks, pp. 1900-1907, Atlanta, GA, USA, June 2009.

[11] Y. Zhang, N. Xu, and Q. Ji, "Study on the application of the hierarchical fuzzy neural network in the fault diagnosis of the asynchronous motor," in 2010 Sixth International Conference on Natural Computation (ICNC), pp. 651-654, Yantai, China, August 2010.

[12] S. S. Yang, J. Xu, and G. Z. Yao, "Concrete strength evaluation based on fuzzy neural networks," in Proceedings of 2004 International Conference on Machine Learning and Cybernetics, pp. 3344-3347, Shanghai, China, August 2004.
[13] Y. Yang, C. Zhu, and J. Xing, "Application of fuzzy neural network to safety evaluation for oil depot," in 2012 3rd International Conference on System Science, Engineering Design and Manufacturing Informatization (ICSEM), pp. 55-58, Chengdu, China, October 2012.

[14] R. Lovassy, L. Gál, Á. Tóth, L. T. Kóczy, and I. J. Rudas, “Fuzzy Flip-flop based neural networks as a novel implementation possibility of multilayer perceptrons," in 2012 IEEE International Instrumentation and Measurement Technology Conference (I2MTC), pp. 280-285, Graz, Austria, May 2012.

[15] F. Djeffal, M. Chahdi, A. Benhaya, and M. L. Hafiane, "An approach based on neural computation to simulate the nanoscale CMOS circuits: application to the simulation of CMOS inverter," Solid-State Electronics, vol. 51, no. 1, pp. 48-56, 2007.

[16] S. Mohagheghi, R. G. Harley, T. G. Habetler, and D. Divan, "Condition monitoring of power electronic circuits using artificial neural networks," IEEE Transactions on Power Electronics, vol. 24, no. 10, pp. 2363-2367, 2009. 


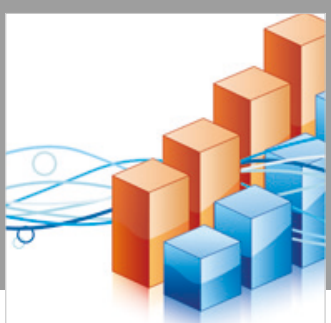

Advances in

Operations Research

\section{-n-m}
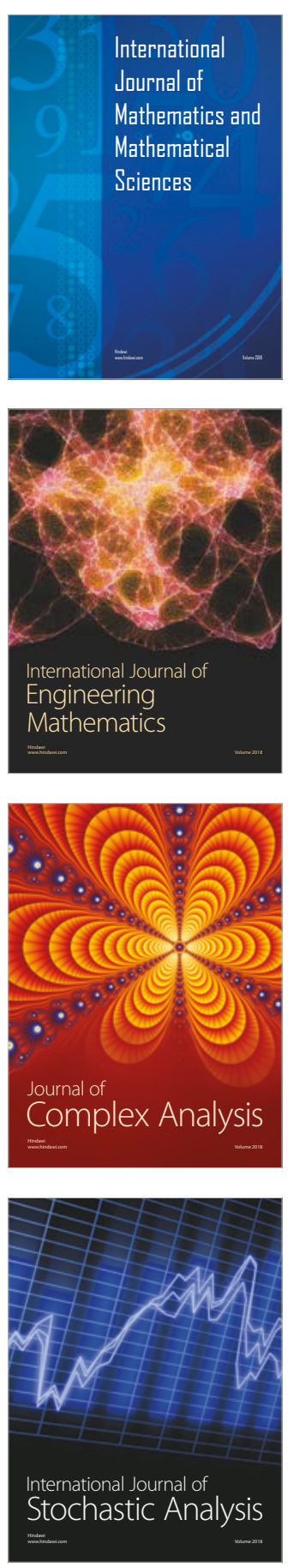
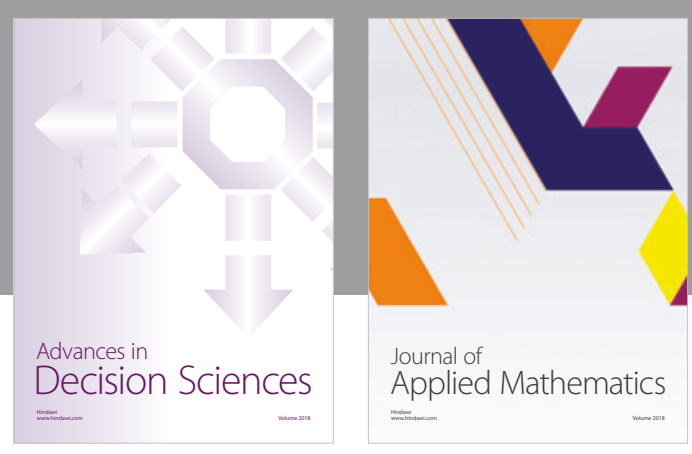

Journal of

Applied Mathematics
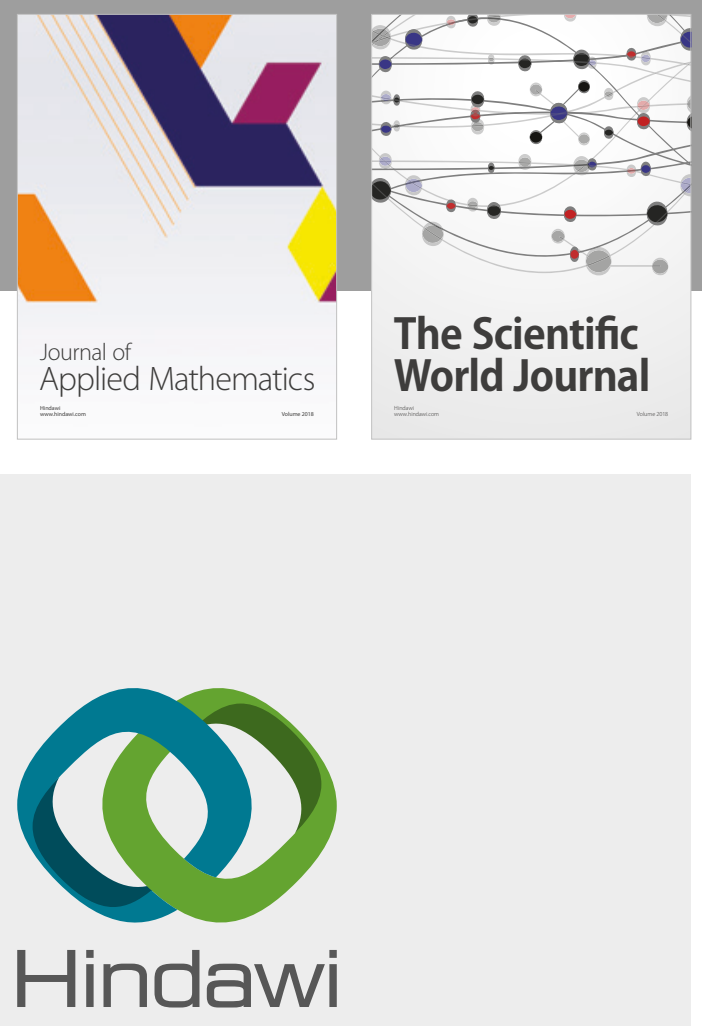

Submit your manuscripts at

www.hindawi.com

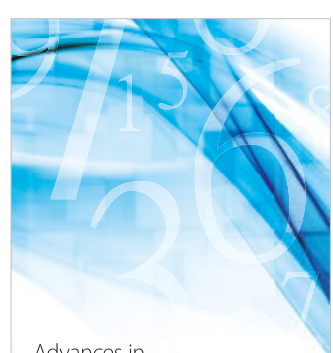

Advances in
Numerical Analysis
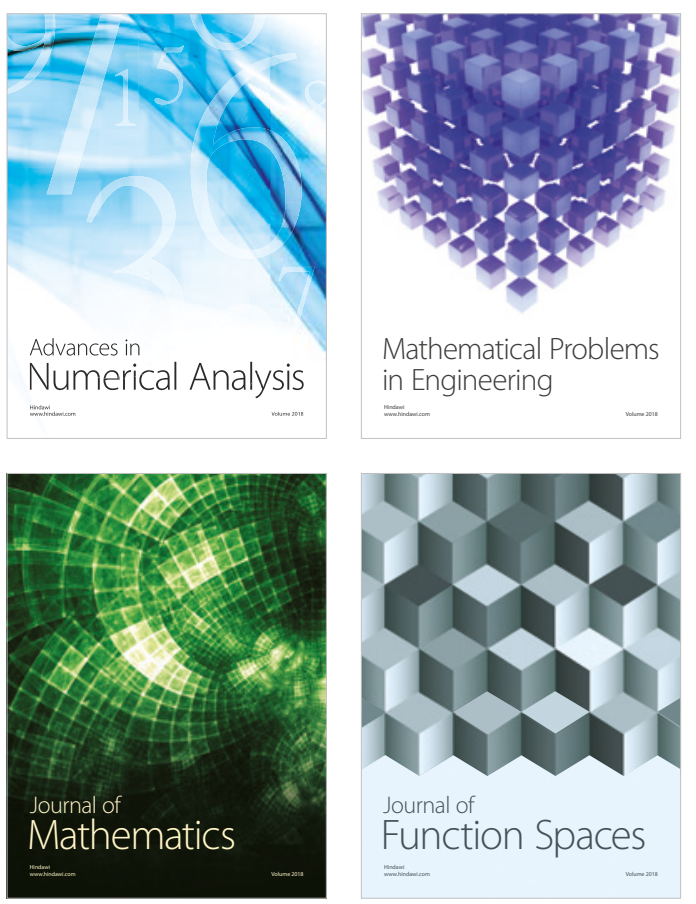

Mathematical Problems in Engineering

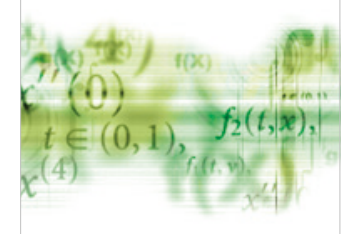

International Journal of

Differential Equations

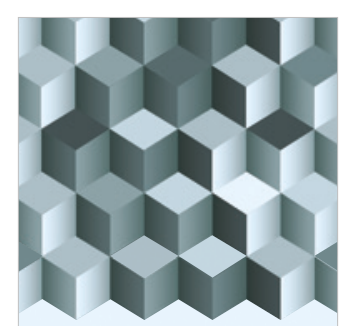

Journal of

Function Spaces
The Scientific

World Journal

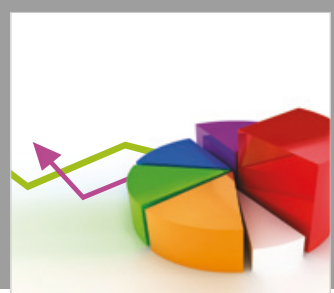

Journal of

Probability and Statistics
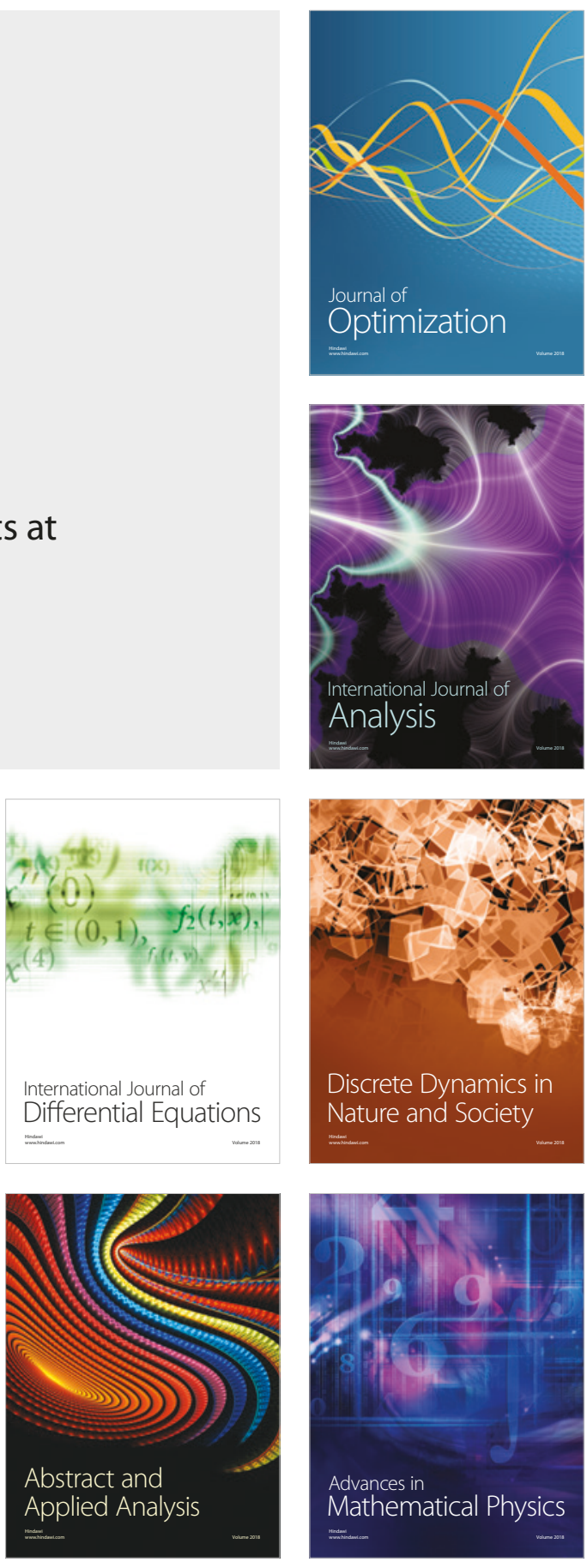